\title{
Susceptibility Vessel Sign on T2*-Weighted Gradient Echo Imaging in Lacunar Infarction
}

\author{
MANSOUR AL-ZGHLOUL, HOLGER WENZ, MÁTÉ MAROS, \\ JOHANNES BÖHME, CHRISTOPH GRODEN and ALEX FÖRSTER
}

Department of Neuroradiology, University Hospital Mannheim, University of Heidelberg, Mannheim, Germany

\begin{abstract}
Background/Aim: In stroke due to large vessel occlusion, thrombotic material can be demonstrated by the susceptibility vessel sign (SVS), a hypointense signal on T2*weighted gradient echo magnetic resonance (GRE) images. In the present study, we evaluated the value of GRE for the detection of perforating artery occlusion in hyperacute lacunar infarction (LI). Materials and Methods: The presence of SVS on GRE in 58 patients with LI who underwent magnetic resonance imaging within 24 hours after symptom onset was analyzed. Results: On diffusionweighted images (DWI), LI was found in the basal ganglia in $17.2 \%$, internal capsule in $25.9 \%$, corona radiata in $19.0 \%$, thalamus in $32.8 \%$, and brainstem in 5.2\%. On GRE a subtle circumscribed hypointense signal at the lower edge of the LI consistent with SVS was detected in 11/58 (19\%) patients. Patients with SVS had larger ischemic lesions on DWI ( $p=0.045)$. Conclusion: SVS on GRE may be useful for detection of perforating artery occlusion in a subset of patients with hyperacute LI.
\end{abstract}

Lacunar infarction (LI) accounts for up to $25 \%$ of acute ischemic strokes (1) and is defined as a small subcortical ischemic infarction in the basal ganglia, thalamus, internal capsule, corona radiata, or brainstem. Classically, the maximal diameter of LI is defined as $<15 \mathrm{~mm}$ but the definition of lacunar lesion size varies widely (2) and more recently, a lesion size $<20 \mathrm{~mm}$ has been introduced in clinical studies (3). LI is usually assumed to be caused by occlusion of a single perforating artery due to lipohyalinosis

This article is freely accessible online.

Correspondence to: Alex Förster, MD, Department of Neuroradiology, Universitätsmedizin Mannheim, Theodor-KutzerUfer 1-3, 68137 Mannheim, Germany. Tel: +49 6213832443, Fax: +496213832165, e-mail: alex.foerster@umm.de

Key Words: Susceptibility vessel sign, T2*-weighted images, lacunar infarction, GRE. or microatheroma $(4,5)$. However, vessel occlusion may also be due to embolism (5), dissection of a small artery (5), or arteriolar wall damage and secondary thrombosis (6). In stroke due to large vessel occlusion, thrombotic material can be demonstrated directly by a hypointense signal on $\mathrm{T} 2 *_{\text {- }}$ weighted gradient echo (GRE) magnetic resonance imaging (MRI) which has been referred to as the susceptibility vessel sign (SVS) (7). In contrast, in LI, vessel occlusion has been observed only occasionally by means of MRI (6).

In the present study, we sought to evaluate the value of GRE for the detection of SVS in perforating artery occlusion in LI, and its frequency in LI.

\section{Materials and Methods}

Patients. We analyzed the data from an earlier study on perfusionweighted imaging (PWI) in LI that included 111 patients with LI. Details of this study have been published elsewhere (8). For the present study, $58(52.2 \%)$ patients with LI and persistent perfusion deficit on PWI were included. MRI was performed in the hyperacute phase of ischemic stroke (within 24 hours after onset of stroke symptoms (9). This study was approved (2013-815R-MA) by the local Institutional Review Board (Medizinische Ethikkommission II der Medizinischen Fakultät Mannheim) and was therefore performed in accordance with the ethical standards laid down in the 1964 Declaration of Helsinki and its later amendments. Patient consent was waived for this analysis by the local Institutional Review Board due to the retrospective nature of the study.

MRI. Magnetic resonance imaging was performed on a 1.5-T MR system (Magnetom Sonata and Magnetom Avanto; Siemens Medical Solutions, Erlangen, Germany) or a 3-T MR system (Magnetom Trio; Siemens Medical Solutions). A standardized stroke MRI protocol was used in all patients including (i) transverse, coronal and sagittal localizing sequences followed by transverse oblique images with a slice thickness of $5 \mathrm{~mm}$ aligned with the inferior borders of the corpus callosum (applied to sequences 2 to 6); (ii) T1-weighted images; (iii) T2-weighted images; (iv) diffusionweighted images (DWI); (v) fluid-attenuated inversion recovery (FLAIR) images; (vi) GRE; (vii) dynamic susceptibility contrast PWI; and (viii) a time-of-flight MR-angiography (TOF-MRA) with a slice thickness of $1 \mathrm{~mm}$. Parameters of GRE are displayed in Table I. Follow-up MRI was performed in 15 (25.9\%) patients. 
Table I. Sequence parameters of T2*-weighted images for magnetic resonance imaging (MRI) scanners used in this study.

\begin{tabular}{lrcr}
\hline Parameter & \multicolumn{3}{c}{ Siemens MRI scanner } \\
\cline { 2 - 4 } & Sonata, 1.5-T & Avanto, 1.5-T & Trio, 3-T \\
\hline FOV, mm ${ }^{2}$ & 240 & 230 & 230 \\
Number of slices $_{\text {Flip angle, }}^{\circ}$ & 24 & 24 & 24 \\
ST, mm & 19 & 20 & 20 \\
TR, ms & 5 & 5 & 5 \\
TE, ms & 670 & 814 & 620 \\
\hline
\end{tabular}

FOV: Field of view, ST: slice thickness, TR: repetition time, TE: echo time.

MRI analysis. Acute ischemic lesions in the basal ganglia, thalamus, internal capsule, corona radiata, or brainstem were classified as LI attributable to single perforating artery occlusion if the maximal diameter was $20 \mathrm{~mm}$ on DWI. Maximal ischemic lesion diameter on DWI as well as maximal diameter of the perfusion deficit on PWI was measured by use of OsiriX (Pixmeo SARL, Bernex, Switzerland), a multidimensional image navigation and display software (10). The SVS was defined as a hypointense signal on GRE at the lower edge of the LI on DWI. GRE were analyzed by two independent raters (M.A. and A.F., both with more than 10 years of experience in neuroimaging) blinded to the other MRI sequences and clinical information. Cases with discrepancies were re-reviewed by both readers and discussed until a consensus was reached.

Statistical analysis. All statistical analyses were performed using Statistical Product and Service Solutions (SPSS) statistics for Windows (Release 17.0; SPSS, Chicago, IL, USA). Comparison between patients with and without SVS was performed using chisquare tests and the Mann-Whitney $U$-test, as appropriate. All statistical analyses were performed with a 0.05 level of significance.

\section{Results}

Overall, $58(52.3 \%)$ patients were included in the analysis. The median age was $69(\mathrm{IQR}=57.75-77)$ years and 27 $(46.6 \%)$ patients were male.

DWI demonstrated an acute ischemic infarction in the basal ganglia in $17.2 \%$, internal capsule in $25.9 \%$, corona radiata in $19.0 \%$, thalamus in $32.8 \%$, and brainstem in $5.2 \%$ with a median diameter of $10.15(\mathrm{IQR}=7.9-13) \mathrm{mm}$.

PWI showed circumscribed hypoperfusion in all patients, with a median diameter of $9.0(\mathrm{IQR}=5.6-12) \mathrm{mm}$. In 40/58 (69.0\%) patients, the ischemic lesion on DWI was larger than the corresponding perfusion deficit on PWI, while in the remainder, the perfusion deficit on PWI was larger than the ischemic lesion on DWI.

On GRE, a subtle circumscribed hypointense signal at the lower edge of the LI consistent with SVS was detected in
11/58 (19\%) patients (for an example, see Figure 1). More pronounced SVS was not demonstrated in any of the patients. On T1-and T2-weighted images, no corresponding signal alterations were observed.

Patients with SVS had larger ischemic lesions on DWI [13.0 mm (IQR=9.4-15.5) vs. $10.0 \mathrm{~mm}(\mathrm{IQR}=7.6-12.8)$, $p=0.045]$ whereas the size of the corresponding perfusion deficits did not differ significantly [7.7 (IQR=7.2.-12.7) $\mathrm{mm}$ vs. $9.0(\mathrm{IQR}=5.2-11.9) \mathrm{mm}, p=0.5]$. Anatomical localization did not differ significantly $(p=0.8)$ between patients with and without SVS. In three patients with LI with SVS, follow-up MRI were available and demonstrated disappearance of the SVS in all of these patients.

\section{Discussion}

In the present study, we investigated the incidence and distribution of SVS in LI for the first time in the hyperacute phase. To date, all available articles regarding SVS in acute ischemic stroke focused on stroke due to large vessel occlusion, such as of the middle cerebral artery, internal carotid artery, or basilar artery $(7,11,12)$. Presence of SVS in posterior cerebral artery occlusion has also been reported anecdotally $(7,13)$. In contrast, perforating artery occlusion in LI has been observed only occasionally in the subacute and chronic phase by means of MRI using conventional MRI sequences as well as GRE (6). To our knowledge, no systematic evaluation regarding the value of GRE for the detection of SVS in hyperacute LI in a larger patient population has been published. The present study describes two essential findings regarding SVS in patients with LI: GRE is capable of displaying SVS in LI in the acute setting, and may be observed in up to $20 \%$ of patients with LI, however, the findings are subtle and may easily be missed.

In 2001, Wardlaw and colleagues reported on the appearance of the presumably occluded perforating artery in a small case series $(n=9)$ of patients with LI in the subacute or chronic phase. They described a linear decreased signal intensity on T2-weighted images and GRE, and increased signal intensity on T1-weighted images in the center or at the edge of LI (6). In comparison to the present study, several differences should be noted: Firstly, the initial MRI was performed in the subacute or even chronic phase of ischemic stroke ( 7 to 58 days after onset of stroke symptoms). Secondly, vessel occlusion was not verified by means of MRI nor pathohistologically. Thirdly, as the authors stated themselves, in some cases the described lesion was wider than should be expected for the diameter of a perforating artery. This might be explained by an initially ectatic perforating artery, thrombus expansion, as well as by leakage of blood in the arterial wall or perivascular space. In the present study, all patients underwent MRI in the hyperacute phase and persistent vessel pathology was demonstrated by 


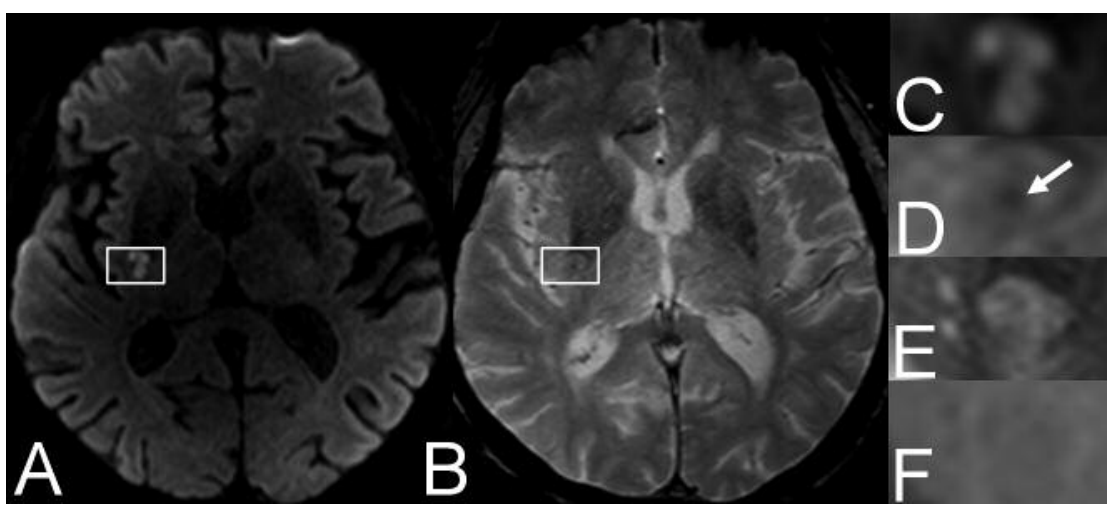

Figure 1. Magnetic resonance imaging of an 83-year-old patient, showing lacunar infarction in the right putamen on diffusion-weighted images (DWI) (A) and corresponding susceptibility vessel sign (SVS) on T2*-weighted gradient echo magnetic resonance (GRE) images (B) images. Magnification of the ischemic lesion on DWI (C) and of SVS on T2*-weighted GRE images $(D)$. T2-weighted $(E)$ and T1-weighted (F) images showed no signal alterations corresponding to SVS.

a perfusion deficit on PWI corresponding to the ischemic lesion on DWI. While the high rate of inverse mismatch (DWI>PWI) might appear counterintuitive on first glance, this might possibly be explained at least partly by the small lesion sizes as well as by the presence of a so-called hidden mismatch due to analytical methods as has been demonstrated in large vessel occlusion (14). Nevertheless, since the underlying vascular lesion could only be determined by neuropathological examination, some uncertainty remains regarding the final diagnosis.

In contrast to the observations of Wardlaw and colleagues, we found only tiny, circumscribed dots of decreased signal intensity at the edge of LI consistent with SVS in a subset of patients but never more extensive changes following the presumed course of a perforating artery. In all patients with follow-up MRI, these lesions resolved completely in the clinical course. Thus, the question arises whether the impressive MRI findings documented in the earlier study demonstrated secondary pathological changes in the course of LI rather than the primary underlying vascular pathology.

Since it has been shown that SVS is more frequently observed in patients with acute ischemic stroke due to cardioembolism (15). This might be of interest for further classification of stroke etiology in patients with small subcortical ischemic infarctions. Diagnosis of LI is usually based on the clinical presentation, stroke morphology and localization on MRI, as well as exclusion of competing etiologies such as cardioembolism or large vessel disease (16). Nevertheless, it has been reported that LI may also occur as a result of embolism (5). In these cases, GRE might be useful in detecting SVS possibly indicating a cardioembolic etiology. Consequently, evidence of SVS could lead to further diagnostic measures with regard to a cardiac source of emboli.
The presents study has some limitations. Firstly, this was a retrospective study of moderate size. However, to our knowledge, this is the first study focusing exclusively on SVS in patients with LI in the hyperacute phase. Secondly, the study was performed with different MRI scanners and different imaging sequences. However, the MRI sequences, especially GRE, were customized for optimal comparability in daily clinical routine and consequently were generally comparable. Thirdly, the hospital-based retrospective study design used might have caused several types of bias and statistical errors such as selection bias, sample bias, or image-based selection bias. Finally, another interpretation of the observed areas with hypointense signal in LI on GRE might be hemorrhagic transformation of the ischemic lesion. However, as secondary hemorrhage in LI is quite rare overall, it seems even more unlikely in our cases as we only included patients with hypoperfusion on PWI indicating persisting vessel occlusion.

\section{Conclusion}

SVS on GRE may be used for detection of acute perforating artery occlusion in LI. However, with regard to the subtle circumscribed findings, this should be confirmed in larger studies, preferably using susceptibility-weighted imaging, which has higher sensitivity for the detection of intraluminal thrombus. Furthermore, future studies should focus on the possible association of SVS in LI with cardiac sources of emboli.

\section{References}

1 Bamford J, Sandercock P, Dennis M, Burn J and Warlow C: Classification and natural history of clinically identifiable subtypes of cerebral infarction. Lancet 337: 1521-1526, 1991. 
2 Potter GM, Marlborough FJ and Wardlaw JM: Wide variation in definition, detection, and description of lacunar lesions on imaging. Stroke 42: 359-366, 2011.

3 Benavente OR, White CL, Pearce L, Pergola P, Roldan A, Benavente MF, Coffey C, McClure LA, Szychowski JM, Conwit R, Heberling PA, Howard G, Bazan C, Vidal-Pergola G, Talbert R and Hart RG: The Secondary Prevention of Small Subcortical Strokes (SPS3) study. Int J Stroke 6: 164-175, 2011.

4 Fisher CM: The arterial lesions underlying lacunes. Acta Neuropathol 12: 1-15, 1968.

5 Fisher CM: Lacunar strokes and infarcts: a review. Neurology 32: 871-876, 1982.

6 Wardlaw JM, Dennis MS, Warlow CP and Sandercock PA: Imaging appearance of the symptomatic perforating artery in patients with lacunar infarction: occlusion or other vascular pathology? Ann Neurol 50: 208-215, 2001.

7 Assouline E, Benziane K, Reizine D, Guichard JP, Pico F, Merland JJ, Bousser MG and Chabriat H: Intra-arterial thrombus visualized on $\mathrm{T}^{*}$ gradient echo imaging in acute ischemic stroke. Cerebrovasc Dis 20: 6-11, 2005.

8 Förster A, Mürle B, Böhme J, Al Zghloul M, Kerl HU, Wenz H and Groden C: Perfusion-weighted imaging and dynamic 4D angiograms for the estimation of collateral blood flow in lacunar infarction. J Cereb Blood Flow Metab 36: 1744-1754, 2016.

9 Allen LM, Hasso AN, Handwerker J and Farid H: Sequencespecific MR imaging findings that are useful in dating ischemic stroke. Radiographics 32: 1285-1297, 2012.

10 Rosset A, Spadola L and Ratib O: OsiriX: an open-source software for navigating in multidimensional DICOM images. J Digit Imaging 17: 205-216, 2004.

11 Naggara O, Raymond J, Domingo AM, Al Shareef F, Touze E, Chenoufi M, Gerber S, Mellerio C, Zuber M, Meder JF, Mas JL and Oppenheim C: T2* "susceptibility vessel sign" demonstrates clot location and length in acute ischemic stroke. PLoS One 8: e76727, 2013.
12 Soize S, Batista AL, Rodriguez RC, Trystram D, Tisserand M, Turc G, Serre I, Ben Hassen W, Zuber M, Calvet D, Mas JL, Meder JF, Raymond J, Pierot L, Oppenheim C and Naggara O: Susceptibility vessel sign on $\mathrm{T} 2 *$ magnetic resonance imaging and recanalization results of mechanical thrombectomy with stent retrievers: a multicentre cohort study. Eur J Neurol 22: 967972, 2015.

13 Park MG, Yoon CH, Baik SK and Park KP: Susceptibility vessel sign for intra-arterial thrombus in acute posterior cerebral artery infarction. J Stroke Cerebrovasc Dis 24: 1229-1234, 2015.

14 Ma HK, Zavala JA, Churilov L, Ly J, Wright PM, Phan TG, Arakawa S, Davis SM and Donnan GA: The hidden mismatch: an explanation for infarct growth without perfusion-weighted imaging/diffusion-weighted imaging mismatch in patients with acute ischemic stroke. Stroke 42: 662-668, 2011.

15 Cho KH, Kim JS, Kwon SU, Cho AH and Kang DW: Significance of susceptibility vessel sign on $\mathrm{T} 2 *$-weighted gradient echo imaging for identification of stroke subtypes. Stroke 36: 2379-2383, 2005.

16 Adams HP Jr., Bendixen BH, Kappelle LJ, Biller J, Love BB, Gordon DL and Marsh EE, III: Classification of subtype of acute ischemic stroke. Definitions for use in a multicenter clinical trial. TOAST. Trial of Org 10172 in Acute Stroke Treatment. Stroke 24: 35-41, 1993. 